\title{
EVALUACIÓN ESPACIAL DE SERVICIOS DE EDUCACIÓN INICIAL: LA DENSIFICACIÓN DE LA OFERTA PARA NIÑOS DE TRES AÑOS EN LA ADMINISTRACIÓN NACIONAL DE EDUCACIÓN PÚBLICA (ANEP)
}

\author{
RICHARD ADRIAN DETOMASI ARAUJO ${ }^{1}$, GERMÁN BOTTO NUÑEZ ${ }^{2}$ \\ ${ }^{1}$ Ministerio de Desarrollo Social (MIDES) \\ Av. 18 de Julio 1453, 11200 Montevideo, Uruguay \\ rdetomasi@mides.gub.uy \\ ${ }^{2}$ Facultad de Medicina, Universidad de la República (UdelaR) \\ Av. Gral. Flores 2125, 11800 Montevideo, Uruguay \\ gbotto@fmed.edu.uy
}

\section{RESUMEN}

La Educación Inicial en Uruguay requiere una orientación en la planificación de su oferta basada en criterios de proximidad a los hogares y en la priorización por vulnerabilidad socioeconómica. El objetivo de este trabajo es evaluar la oferta existente y las ampliaciones planteadas, y proponer la creación de nuevos centros para cubrir la demanda no satisfecha. Para esto se modeló la distribución de la población objetivo a nivel de punto para todo el país y se aplicó una metodología de asignación óptima. En términos absolutos la oferta debería ser suficiente para cubrir la demanda. Sin embargo, la diferencia entre la distribución espacial de la población objetivo y la de los servicios hace que la oferta sea insuficiente y que, de aplicarse los principios rectores en cuanto a distancia y tamaño de los grupos, un $35 \%$ de los cupos previstos en las ampliaciones no sean utilizados.

Palabras clave: Localización óptima, Administración Nacional de Educación Pública, Uruguay, Polígonos de Voronoi, Clusterización espacial. 
Detomasi Araujo R. A., Botto Nuñez G. (2017): “Evaluación espacial de servicios de educación inicial: la densificación de la oferta para niños de tres años en la Administración Nacional de Educación Pública (ANEP)”, GeoFocus (Artículos), $n^{o}$ 20, p. 49-61. ISSN: 1578-5157 http://dx.doi.org/10.21138/GF.508

SPATIAL ASSESMENT OF PRESCHOOL EDUCATIONAL SERVICES: DENSIFICATION OF NATIONAL ADMINISTRATION OF PUBLIC EDUCATION'S OFFER FOR THREE YEARSOLD CHILDREN

\section{ABSTRACT}

The pre-school education in Uruguay needs a reorientation in its offer planning, based upon criteria of proximity to target homes and socio-economical vulnerability prioritization. The objective of this work was to assess the coverage of the current offer, the proposed capacity's increases and to propose the creation of new centers to cover the unsatisfied demand. To achieve this, the population distribution was modeled to a point level and an optimal assignment algorithm was applied. While in absolute numbers the current offer should be enough to cover the demand, the differences in spatial distributions made the offer inadequate to satisfy the demand and that, if distance and group size criteria are applied, $35 \%$ of the proposed capacity's increases would not be used.

Keywords: Optimal location, National Administration of Public Education, Uruguay, Voronoi's Tessellation, Spatial Clustering.

\section{Introducción}

En Uruguay, la oferta pública de educación inicial para niños de 3 años se realiza a través de los Centros de Atención a la Infancia y la Familia (CAIF), los Centros Diurnos (CD) del Instituto del Niño y el Adolescente de Uruguay (INAU), y los grupos de 3 años atendidos por la Administración Nacional de Educación Pública (ANEP). En el marco de la implementación del Sistema Nacional Integrado de Cuidados (SNIC) de Uruguay, los encargados de la Educación Inicial Pública coinciden en la necesidad de una evaluación en la planificación de su oferta, basando la misma en criterios de proximidad a los hogares y en la priorización de la cobertura hacia los hogares socioeconómicamente más vulnerables. Tradicionalmente, las proyecciones de ampliación de los servicios educativos en Uruguay se basaron más en una evaluación cualitativa de la demanda social, que en la aplicación un marco de referencia analítico que permitiera replicar y evaluar los criterios de decisión (UNESCO, 2011). Ante una expansión del sistema público, impulsada y financiada por el SNIC, se entendió pertinente optimizar este proceso de ampliación, para mejorar la accesibilidad a los servicios educativos para la población y para optimizar el uso de los recursos económicos.

En este trabajo se toma el caso de los centros ANEP y su propuesta de ampliación de la oferta para el quinquenio 2015-2019. El objetivo es proporcionar una cobertura universal a los niños de 3 años, por lo que esta propuesta plantea principalmente cubrir la demanda a partir de la infraestructura existente, y en los casos necesarios generar nuevos grupos.

La delimitación de la vulnerabilidad social en zonas y situaciones heterogéneas del tejido social, reformuló en parte la visión tradicional de políticas de asistencia a la pobreza que se concentraba en la determinación de una frontera precisa, basada en la línea de ingresos. En 2008, 
Detomasi Araujo R. A., Botto Nuñez G. (2017): “Evaluación espacial de servicios de educación inicial: la densificación de la oferta para niños de tres años en la Administración Nacional de Educación Pública (ANEP)”, GeoFocus (Artículos), $n^{o}$ 20, p. 49-61. ISSN: 1578-5157 http://dx.doi.org/10.21138/GF.508

como parte de un convenio entre la Universidad de la República y el Ministerio de Desarrollo Social, se desarrolló el Índice de Carencias Críticas (ICC) como una herramienta adecuada a la hora de definir la vulnerabilidad multidimensional (DAES-MIDES, 2015). Este índice tiene en cuenta, entre otros factores, los ingresos del hogar, las condiciones habitacionales y del entorno, la composición del hogar, las características de sus integrantes y la situación sanitaria (Ley 18.277, art. 2). El ICC es utilizado actualmente como orientador de las políticas sociales del Ministerio de Desarrollo Social (MIDES) de Uruguay para la caracterización de la población elegible para sus políticas de transferencia monetaria (Amarante et al., 2008; DAES-MIDES, 2015).

El ICC sintetiza información de los hogares para determinar su nivel de vulnerabilidad, sin incluir directamente el ingreso (DAES-MIDES, 2015). El valor del índice es un indicador de la vulnerabilidad socioeconómica del grupo familiar: a mayor valor del ICC, mayor nivel de vulnerabilidad del grupo familiar. A partir del cálculo del ICC (rango [0-1]) se establecen tres umbrales que definen cuatro grupos de población. Estos grupos son la guía para la inclusión de las familias en los diferentes programas de transferencias monetarias. El menos crítico de los umbrales es utilizado para la inclusión de los núcleos familiares en el programa de Asignaciones FamiliaresPlan de Equidad (AFAM-PE) (DAES-MIDES, 2015).

La equidad espacial se plasma en la búsqueda de homogeneizar la accesibilidad espacial de la demanda potencial a los centros, y de esta manera evitar penalizar aquellos puntos de demanda que, por su aislamiento, no tienen una buena conexión con la red (Bosque y Moreno, 2004). Pero la equidad es un criterio disputado, tanto en la forma de medirlo como en la misma conveniencia de considerarlo para las decisiones de localización (Matallana, 2010). Dependiendo de cada teoría ética que defiende la búsqueda de la equidad, la igualdad basal (Sen, 1996) se prioriza en distintos espacios (ingresos, utilidades, derechos, libertades u otros, según las ideas de justicia que se manejen). Para esta investigación, planteada desde la teoría de la localización de facilidades públicas, la equidad fue trabajada en relación con la accesibilidad (relación oferta/demanda, distancia y capacidad instalada de los centros) utilizando el ICC como aproximación a los factores sociales, pero sin tener en cuenta factores políticos u otros que inciden en el bienestar social, ya que forman parte del territorio y de un campo más grande que es el espacio social.

La localización óptima de servicios implica encontrar la mejor ubicación para un servicio en relación con una serie de criterios establecidos a priori (Bosque y Moreno, 2004). Desde el punto de vista geográfico la localización de servicios debe respetar principios tales como la eficiencia espacial, la justicia espacial, la efectividad y la gestión (Ramírez y Bosque, 2001). La representación espacial de la demanda puede realizarse como una population surface (Bracken y Martin, 1989; 1995) o a partir de bases catastrales (Maantay et al., 2007), buscando una mayor desagregación espacial del dato. Enfrentando la discusión sobre el Modifiable Area Unit Problem (MAUP) (Li et al., 2007), la modelación estará siempre sujeta al detalle de agregación con el que son recabados y almacenados los datos censales. En el caso de Uruguay, la información censal se encuentra disponible a nivel de segmentos censales, que representan la menor unidad geoestadística no protegida bajo secreto censal (Ley 16.616 y Ley 18.331). Esta unidad es equivalente a una manzana en el caso de áreas urbanas consolidadas, aunque esto no se cumple para áreas no consolidadas (zonas rurales), que varían ampliamente en forma, tamaño y distribución interna de la población a lo largo del país (INE, s/f). 
Detomasi Araujo R. A., Botto Nuñez G. (2017): “Evaluación espacial de servicios de educación inicial: la densificación de la oferta para niños de tres años en la Administración Nacional de Educación Pública (ANEP)”, GeoFocus (Artículos), $n^{o}$ 20, p. 49-61. ISSN: 1578-5157 http://dx.doi.org/10.21138/GF.508

La reducción de la escala de la información censal, sin acceder a datos sensibles, implica la utilización de información accesoria que dé cuenta de la densidad de población dentro de cada segmento censal. Casos extremos serían por ejemplo los que rasterizan el dato de población para que pueda ser reasignado a la escala en la que se trabaje (Balk et al. 2006; CIESIN, 2016), pero de todos modos esta reducción de la escala mantiene la continuidad en el espacio del dato, mientras que la estrategia que se plantea en este documento se propone vectorizar los datos a nivel de puntos, y a través de herramientas de aleatorización garantizar el secreto estadístico debido.

Existen al menos cuatro fuentes de datos en Uruguay que permiten inferir la distribución de la población a nivel de puntos y que pueden combinarse con información censal para reducir la escala de esta última. Estas fuentes de datos son la distribución de contadores domiciliarios de energía eléctrica y agua corriente, las viviendas rurales georreferenciadas durante el último censo nacional y la ubicación de viviendas obtenida a partir de las visitas que realiza el MIDES para el seguimiento de sus programas. Cada una de las fuentes mencionadas tiene una cobertura diferente, pero si se construye una capa de puntos que represente a todas las viviendas del país, es posible distribuir entre esas viviendas la información censal a nivel de segmentos.

El objetivo entonces de este trabajo es evaluar la oferta existente, la ampliación de oferta propuesta por ANEP, y realizar una propuesta de proyección de nuevos centros. El interés principal radica en incorporar la información geográfica de los problemas sociales para mejorar las posibilidades de abordaje, ampliando y precisando las estrategias de políticas.

\section{Materiales, datos y métodos}

\subsection{El caso de estudio}

Este trabajo pretende estudiar la distribución de los cupos de educación inicial en Uruguay. Para esto se enfocó en el funcionamiento del sistema en el año 2014 y en la planificación para el quinquenio 2015-2019. El alcance espacial es nacional, ya que se pretendió dar una solución para la planificación del sistema por parte de la ANEP. El encuadre temporal se justifica en la disponibilidad de los datos para el modelado y los plazos de los programas gubernamentales en educación.

\subsection{Fuentes de datos}

Para el modelado de la demanda potencial se construyó una capa de información con la distribución de los niños de 3 años en todo el país. De la información publicada a nivel de segmento censal de los censos 2011 (INE, 2012, Cabella et al., 2012) se obtuvo la cantidad de niños de tres años y el tipo de asistencia a centros educativos. La proporción de hogares elegibles para el programa AFAM-PE se obtuvo a partir del cálculo de un proxy del ICC a partir de información censal (Bai et al., 2014).

En definitiva, la población total de Uruguay, según los censos 2011 del INE, era de 3.286.314 de habitantes, de los cuales 34.998 eran niños de 3 años que no asistían a centros de educación privados o integraban hogares elegibles para AFAM-PE. (Tabla 1). 
Detomasi Araujo R. A., Botto Nuñez G. (2017): “Evaluación espacial de servicios de educación inicial: la densificación de la oferta para niños de tres años en la Administración Nacional de Educación Pública (ANEP)”, GeoFocus (Artículos), $n^{o}$ 20, p. 49-61. ISSN: 1578-5157 http://dx.doi.org/10.21138/GF.508

Tabla 1. Desagregación de la demanda de asistencia en 3 años

\begin{tabular}{|l|c|c|c|}
\hline & Cantidad & $\begin{array}{c}\text { Porcentaje (sobre } \\
\text { total de 3 años) }\end{array}$ & $\begin{array}{c}\text { Porcentaje (sobre no } \\
\text { asistentes a privados) }\end{array}$ \\
\hline Total & 44.553 & $100 \%$ & - \\
\hline En hogares AFAM-PE & 25.632 & $58 \%$ & - \\
\hline $\begin{array}{l}\text { Que no asisten a centros } \\
\text { privados }\end{array}$ & 33.241 & $75 \%$ & $71 \%$ \\
\hline $\begin{array}{l}\text { Que no asisten a centros } \\
\text { privados, en hogares AFAM-PE }\end{array}$ & 23.446 & $53 \%$ & \\
\hline
\end{tabular}

Fuente: elaboración propia en base a datos del Censo 2011 (INE)

Para construir una capa con la distribución de viviendas del país se combinaron cuatro fuentes de datos complementarias: viviendas rurales relevadas por los Censos de 2011, ubicación de contadores de energía eléctrica, ubicación de contadores de servicio de agua corriente y visitas georreferenciadas de trabajo de campo del MIDES. Para mantener la confidencialidad de los datos, en todos los casos se registró únicamente el par de coordenadas (x, y) para cada hogar desvinculándolo de toda la información de registro.

La información de matrícula actual (diciembre/2014 para ANEP y marzo/2015 para INAU) y la ubicación de cada servicio socioeducativo se obtuvieron a través del SIIAS y a partir de la información que provee al sistema cada organismo interviniente (ANEP, CAIF e INAU). La ampliación de los cupos prevista para el quinquenio 2015-2019 fue provista directamente por ANEP. Y como se detalla en la Tabla 2, el sistema educativo público actualmente ofrece 36.166 cupos para esta edad distribuidos entre el programa CAIF y CD INAU.

Tabla 2. Distribución de los cupos existentes, según el prestador de servicio para la población de 3 años (total país)

\begin{tabular}{|c|c|c|c|c|c|}
\hline & CAIF & $\begin{array}{c}\text { CD } \\
\text { INAU }\end{array}$ & $\begin{array}{c}\text { ANEP } \\
\text { existentes }\end{array}$ & $\begin{array}{c}\text { ANEP } \\
\text { proyectados }\end{array}$ & Total \\
\hline Cupos Existentes & $\begin{array}{c}15.778 \\
(43,6 \%)\end{array}$ & $\begin{array}{c}600 \\
(1,7 \%)\end{array}$ & $\begin{array}{c}11.688 \\
(32,3 \%)\end{array}$ & $\begin{array}{c}8.100 \\
(22,4 \%)\end{array}$ & $\begin{array}{c}36.166 \\
(100 \%)\end{array}$ \\
\hline
\end{tabular}

Fuente: elaboración propia en base a ANEP (diciembre/2014) e INAU (marzo/2015)

\subsection{Técnicas de análisis}

A partir de las fuentes de datos de las viviendas, se seleccionó para cada zona censal (mínima unidad geoestadística nacional) (INE, s/f) la fuente de información que presentaba mayor cantidad de registros y se combinaron todas las zonas en una única capa de información.

Dentro de los puntos de viviendas se realizó un sorteo con reposición, por segmento censal, para seleccionar tantas observaciones como niños registrados hay en cada unidad, de acuerdo a la información censal. Una vez seleccionados los puntos correspondientes a los niños de tres años se sorteó al azar la pertenencia de cada hogar modelado a la población elegible para AFAM-PE y la asistencia o no a centros de educación privada, de acuerdo a las proporciones registradas para cada 


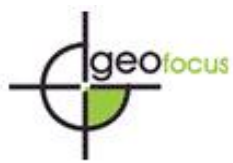

Detomasi Araujo R. A., Botto Nuñez G. (2017): “Evaluación espacial de servicios de educación inicial: la densificación de la oferta para niños de tres años en la Administración Nacional de Educación Pública (ANEP)”, GeoFocus (Artículos), $n^{o}$ 20, p. 49-61. ISSN: 1578-5157 http://dx.doi.org/10.21138/GF.508

segmento censal. Finalmente se consolidó una capa de demanda con los puntos sorteados como elegibles para AFAM-PE y/o no asistentes a centros de educación privada.

Para la construcción de una capa de oferta de servicios se utilizó la ubicación de los servicios actuales de CAIF, ANEP y centros diurnos de INAU junto a la ampliación de servicios propuesta por ANEP para el quinquenio 2015-2019. Se consideró la matrícula actual como un indicador de la capacidad efectiva de cada servicio. Para las ampliaciones se tomó el cupo proyectado como capacidad de cobertura.

Para reorientar la oferta de los centros educativos y cumplir con las restricciones impuestas al análisis se consideró que los cupos actuales se cubrirían con los niños del entorno inmediato de cada centro, específicamente con menores cuyos domicilios estuvieran situados a menos de $1 \mathrm{~km}$ de distancia del centro. Para esto se construyeron polígonos de Voronoi (Okabe et al., 2000) a partir de los centros educativos y se asignó a cada menor al centro más cercano utilizando los polígonos. Una vez identificado el centro más cercano a cada domicilio se calculó la distancia euclídea de cada centro a los domicilios asignados al mismo. La oferta de cupo se cubrió con los $\mathrm{n}_{\mathrm{i}}$ niños más cercanos en el entorno de un kilómetro (siendo $\mathrm{n}_{\mathrm{i}}$ la cantidad de cupos que actualmente cubre). La figura 1 muestra una esquematización del proceso.

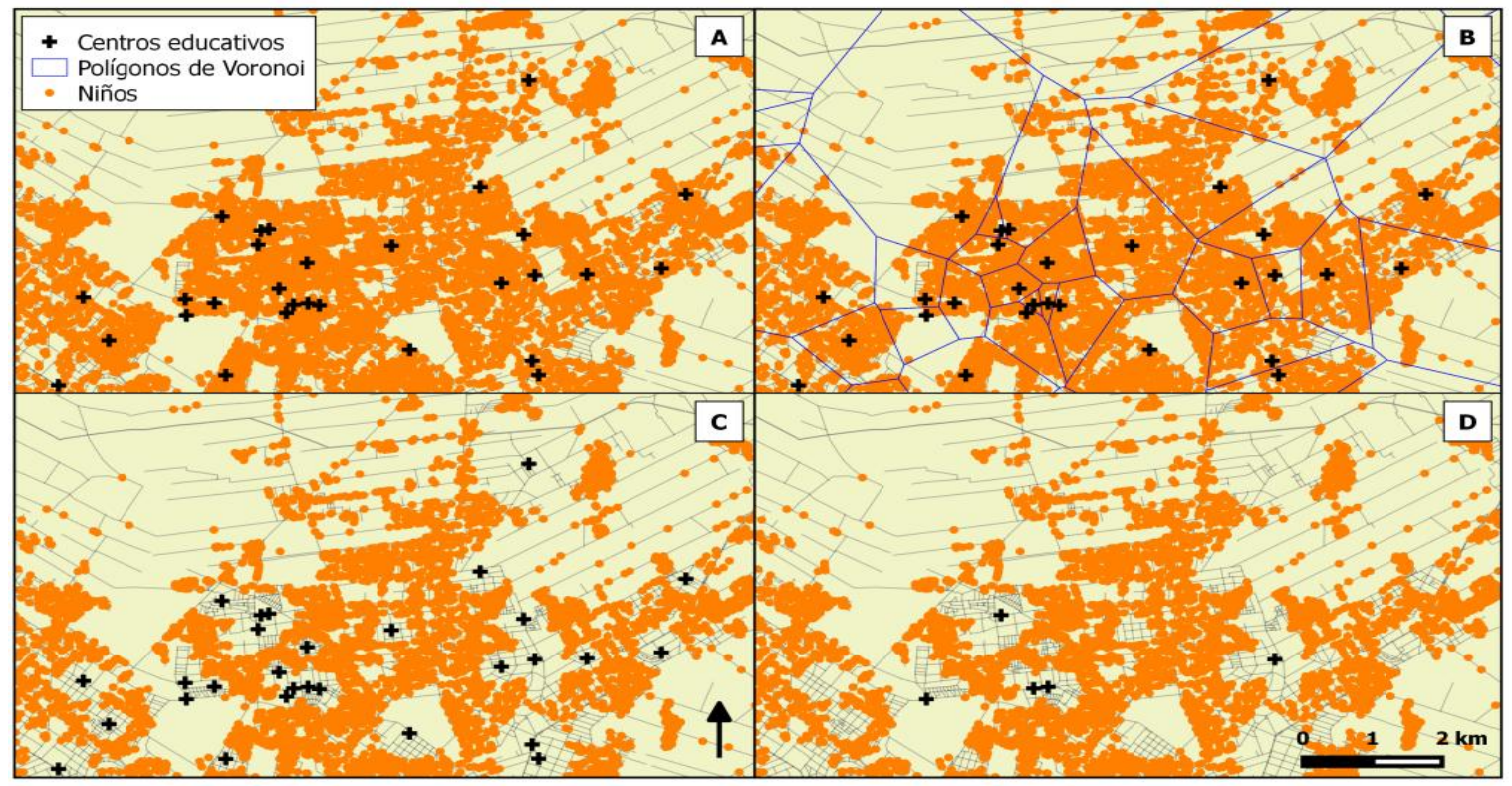

Figura 1. Esquema de un paso del proceso iterativo de asignación de los niños (puntos de demanda) a los centros existentes (puntos de oferta). A: Ubicación de los puntos de demanda y de oferta. B: Polígonos de Voronoi generados a partir de los centros, utilizados para asignar cada domicilio al centro educativo más cercano. C: Eliminación de los niños considerados cubiertos por cada centro existente. D: Eliminación de los centros cuyo cupo fue cubierto (modificado de Botto y Detomasi, 2015).

El proceso se repitió cuatro veces, ya que en algunos sitios la configuración muy agrupada de centros no permitía cubrir en un solo paso los cupos ofrecidos por todos. Para cada repetición se 
Detomasi Araujo R. A., Botto Nuñez G. (2017): “Evaluación espacial de servicios de educación inicial: la densificación de la oferta para niños de tres años en la Administración Nacional de Educación Pública (ANEP)”, GeoFocus (Artículos), $n^{o}$ 20, p. 49-61. ISSN: 1578-5157 http://dx.doi.org/10.21138/GF.508

eliminaban de las bases para el análisis los centros cuyo cupo estaba cubierto y los niños que habían cubierto esos cupos. Finalmente se realizó una última asignación, pero flexibilizando el criterio de distancia de $1 \mathrm{~km}$ a $5 \mathrm{~km}$, considerando que en zonas suburbanas o periferias urbanas podía aún ser una distancia razonable para la asistencia al centro. Una vez concluida la asignación, aquellos cupos no cubiertos se consideraron perdidos (como imprecisión de focalización espacial de la política actual y la proyectada).

Con los niños no cubiertos por la oferta actual o la proyectada para el quinquenio, se propuso la creación de nuevos centros considerando la agrupación espacial de los menores. Para esto se realizó un proceso iterativo de agrupamiento mediante clusters de k-medias (Gotelli y Ellison, 2004) considerando únicamente las coordenadas planas como variable de agrupamiento. Para determinar el número de grupos $(\mathrm{k})$ de cada etapa del proceso se utilizó el cociente entre las observaciones a asignar $(\mathrm{N})$ y el tamaño de los grupos a crear $\left(\mathrm{n}_{\mathrm{i}}\right)$ :

$$
k=\frac{N}{n_{i}}
$$

Los nuevos centros se ubicaron en los centros medianos (intersección de la mediana de las coordenadas) de cada grupo. Una vez determinada la ubicación del centro a crear, se asignaron a este los niños más cercanos siguiendo la misma metodología utilizada para la asignación a centros existentes. Los niños no cubiertos en cada paso pasaban a conformar la demanda para la siguiente iteración. Se crearon tres tipos de centros bajo esta modalidad: con grupos de 25 niños (5 iteraciones), con grupos de 12 niños (2 iteraciones) y centros atípicos que nuclearon niños dispersos que no pueden ser cubiertos con las modalidades anteriores ( 2 iteraciones). Los centros atípicos eliminan los criterios de tamaño de grupo, primero, y de distancia, luego, considerando dentro a todos los niños cubiertos asignados al centro. Para la determinación de $\mathrm{k}$ en estas últimas iteraciones, se consideró $\mathrm{n}_{\mathrm{i}}=12$.

El proceso de asignación de la demanda a los centros existentes fue adaptado de Botto y Detomasi (2015). Todo el procesamiento de datos se programó utilizando R 3.1.0 (R Core Team, 2015), utilizando la librería EPP v0.1.0, accesible actualmente para uso público (Detomasi y Botto, 2017).

\section{Descripción y análisis de resultados}

Los resultados obtenidos tras la aplicación de la metodología antes descrita mostraron que, una vez distribuidos los niños entre los centros existentes y los proyectados por ANEP, hay 5.669 cupos no utilizados -que representan un $15,7 \%$ de la oferta- debido a la diferencia entre la distribución espacial de los servicios y de la demanda. Estos cupos serán analizados posteriormente.

El total de niños no cubiertos por la oferta actual o proyectada es lo que se considera como la demanda insatisfecha, para la cual se requiere la construcción de nuevos centros. La diferencia entre la demanda y los cupos utilizados representa 4.501 niños de 3 años. Esta demanda no satisfecha deberá ser cubierta por nuevos centros o correcciones de las aperturas o ampliaciones proyectadas (Tabla 3). 
Detomasi Araujo R. A., Botto Nuñez G. (2017): "Evaluación espacial de servicios de educación inicial: la densificación de la oferta para niños de tres años en la Administración Nacional de Educación Pública (ANEP)”, GeoFocus (Artículos), $n^{o}$ 20, p. 49-61. ISSN: 1578-5157 http://dx.doi.org/10.21138/GF.508

Tabla 3. Distribución de la demanda para la población de 3 años (total país)

\begin{tabular}{|l|c|c|}
\hline & Frecuencia absoluta & Frecuencia relativa \\
\hline Demanda & 34.998 & $100 \%$ \\
\hline Demanda cubierta & 30.497 & $87 \%$ \\
\hline Demanda no satisfecha & 4.501 & $13 \%$ \\
\hline
\end{tabular}

Fuente: elaboración propia en base a procesamiento.

Por su parte, los cupos no utilizados por los centros existentes son evaluados con miras a otras soluciones (i.e. transporte escolar, etc.), mientras que los cupos sobrantes de los centros ANEP proyectados también son expuestos a reorientaciones por parte de la institución, distinguiéndose entre los centros a ampliar y los nuevos (Tabla 4), dadas las implicancias edilicias claramente diferentes.

Tabla 4. Distribución de los cupos existentes, utilizados y no utilizados, según el prestador de servicio, para la población de 3 años (total país)

\begin{tabular}{|c|c|c|c|c|c|}
\hline & CAIF & $\begin{array}{c}\text { CD } \\
\text { INAU }\end{array}$ & $\begin{array}{c}\text { ANEP } \\
\text { Existentes }\end{array}$ & $\begin{array}{c}\text { ANEP } \\
\text { Proyectados }\end{array}$ & Total \\
\hline Cupos existentes & $\begin{array}{c}15.778 \\
(100 \%)\end{array}$ & $\begin{array}{c}600 \\
(100 \%)\end{array}$ & $\begin{array}{c}11.688 \\
(100 \%)\end{array}$ & $\begin{array}{c}8.100 \\
(100 \%)\end{array}$ & $\begin{array}{c}36.166 \\
(100 \%)\end{array}$ \\
\hline Cupos utilizados & 13.933 & 570 & 10.733 & 5.261 & 30.497 \\
& $(88 \%)$ & $(95 \%)$ & $(92 \%)$ & $(65 \%)$ & $(84 \%)$ \\
\hline Cupos no utilizados & 1.845 & 30 & 955 & 2.839 & 5.669 \\
& $(12 \%)$ & $(5 \%)$ & $(8 \%)$ & $(35 \%)$ & $(16 \%)$ \\
\hline
\end{tabular}

Fuente: elaboración propia en base a procesamiento.

En definitiva, el cuadro siguiente (Tabla 5) muestra estas contrapropuestas, y un indicador de consideración por parte de la institución, siendo esta una medida por lo menos aproximada de la relevancia de esta metodología para la evaluación de ofertas proyectadas:

Tabla 5. Exceso de oferta ANEP para la población de 3 años (total país)
\begin{tabular}{|l|c|c|}
\hline & Centros & Cupos \\
\hline ANEP existentes & 41 & 955 \\
\hline ANEP Ampliaciones proyectadas & 27 & 1.339 \\
\hline ANEP Aperturas proyectadas & 29 & 1.500 \\
\hline Total & 97 & 3.794 \\
\hline
\end{tabular}

Fuente: elaboración propia en base a procesamiento.

Aunque la cobertura proyectada por ANEP aparenta ser suficiente en números absolutos, las restricciones de distancia vuelven muy relativa esta afirmación.

Según el análisis realizado, habría 4.501 niños de 3 años distribuidos por todo el territorio nacional que no estarían cubiertos. A la vez, de los 8.100 cupos proyectados (entre ampliaciones y creación de nuevos centros), 2.839 se pierden por no encontrar niños a cubrir en las cercanías (Tabla 4). Considerando el cupo actual de los centros existentes, se perderían 955 cupos, lo que representa un $8,2 \%$ de la oferta actual. 
Detomasi Araujo R. A., Botto Nuñez G. (2017): "Evaluación espacial de servicios de educación inicial: la densificación de la oferta para niños de tres años en la Administración Nacional de Educación Pública (ANEP)”, GeoFocus (Artículos), $n^{o}$ 20, p. 49-61. ISSN: 1578-5157 http://dx.doi.org/10.21138/GF.508

Para la demanda no cubierta por la oferta actual o proyectada se crearon un total de 201 grupos, 114 de ellos en las dos primeras modalidades (grupos de 12 o 25 niños) y el resto en grupos atípicos (Tabla 6). Aproximadamente el $60 \%$ de la demanda no cubierta requeriría de grupos atípicos, lo que muestra la dispersión de esta población.

Tabla 6. Distribución de los cupos generados entre los tipos de grupos a crear

\begin{tabular}{|l|c|c|}
\hline \multicolumn{1}{|c|}{ Tipo } & Grupos & Cupos \\
\hline De 25 niños & 37 & 925 \\
\hline De 12 niños & 77 & 924 \\
\hline Atípicos & 87 & 2.652 \\
\hline Total & 201 & 4.501 \\
\hline
\end{tabular}

Fuente: elaboración propia en base a procesamiento.

Finalmente, como muestra la Figura 2, por un lado, las concentraciones de niños de 3 años que no estarían cubiertos por la oferta existente ni la proyectada, rodean varios de los centros que se indican como cupos sin cubrir por los niños cercanos a ellos, poniendo a discusión lo estricto del criterio de distancia utilizado. Mientras que, por otro lado, muestra claramente la distribución por la cual más de la mitad de los niños de 3 años no cubiertos forman los 87 centros atípicos indicados en la Tabla 6.
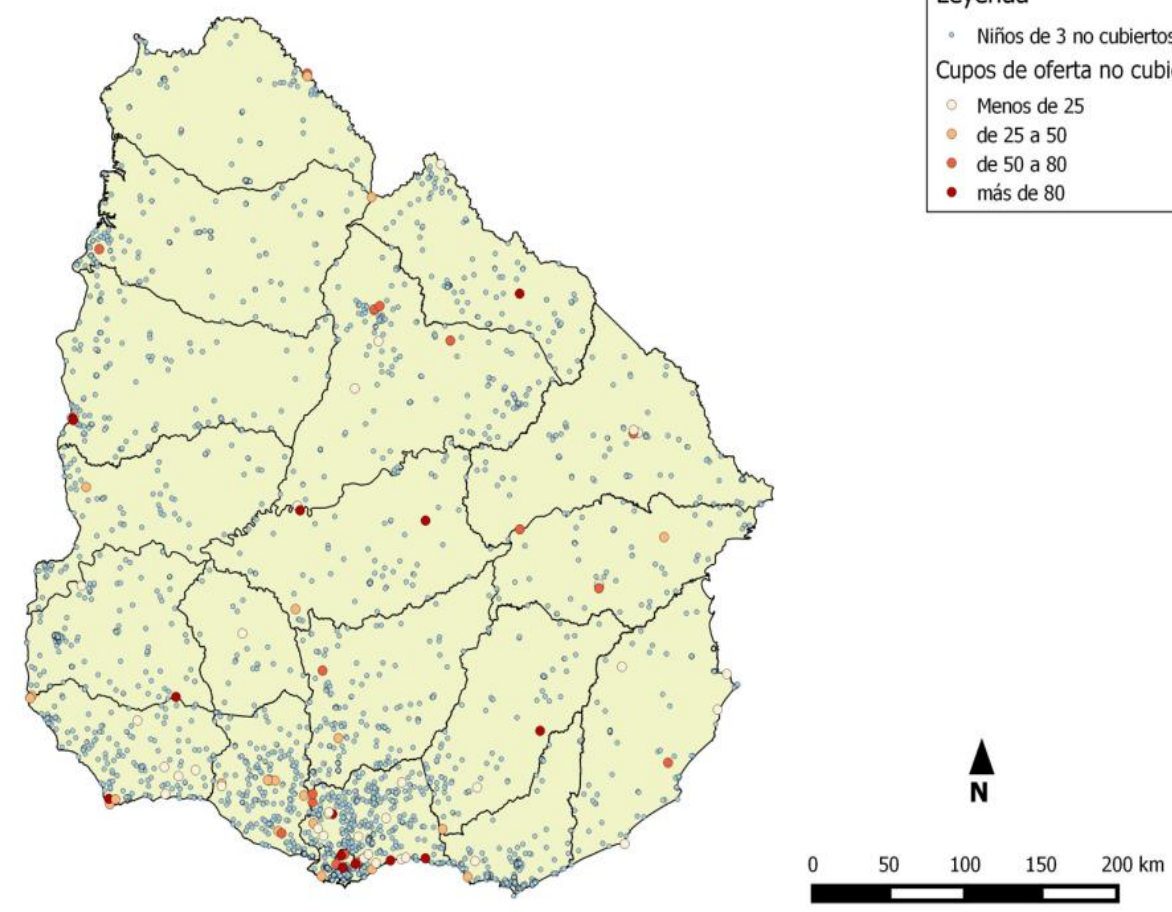

Figura 2. Distribución de niños de 3 años no cubiertos por la oferta existente ni proyectada por ANEP y cupos no utilizados según análisis de centros existentes o proyectados por ANEP.

(C) Los autores 
Detomasi Araujo R. A., Botto Nuñez G. (2017): “Evaluación espacial de servicios de educación inicial: la densificación de la oferta para niños de tres años en la Administración Nacional de Educación Pública (ANEP)”, GeoFocus (Artículos), $n^{o}$ 20, p. 49-61. ISSN: 1578-5157 http://dx.doi.org/10.21138/GF.508

\section{Discusión y conclusiones}

La propuesta original de ampliación de servicios analizada en este trabajo fue desarrollada en principio por la propia institución, atendiendo a parámetros principalmente infraestructurales: posibilidad de ampliaciones edilicias en los centros existentes, o listas de espera para el ingreso pero que solo consideraban la oferta en su momento y en funcionamiento, etc. Reestudiar esa propuesta de ampliación del servicio desde una perspectiva de localización óptima permitirá evaluar el impacto potencial de las políticas y optimizar su aplicación.

El análisis realizado muestra, por un lado, que de implementarse tal cual el plan original, un porcentaje importante de los cupos generados (a través de ampliaciones de centros o creaciones de centros nuevos), quedaría inutilizado bajo los criterios de accesibilidad espacial planteados. Esto quiere decir que, o bien los cupos no serían utilizados o, más probablemente, los cupos serían utilizados por menores que deberían desplazarse más allá de lo que se considera óptimo en la propuesta, generando una situación de inequidad espacial. La metodología propuesta en este trabajo se basa en dos puntos fundamentales: una definición de "accesibilidad óptima" y las fuentes de información utilizadas. La incorporación de una definición espacialmente explícita de accesibilidad permite objetivar el análisis de equidad espacial. Además, permite realizar evaluaciones de escenarios alternativos.

En el caso de Uruguay, la información a nivel de zona censal no es de acceso público, ya que se encuentra protegida bajo secreto estadístico. Es por eso que se utiliza la distribución de las puestas de servicios que sirven como variable proxy de la distribución de la población. Además, se utiliza información de relevamientos realizados directamente a nivel de hogares, pero preservando el secreto censal a través únicamente del uso del punto (posición) sobre el cual se registró el dato. Este procedimiento permite modelar la distribución espacial de la demanda a nivel de punto, representando adecuadamente la dispersión no uniforme dentro de cada unidad geoestadística, ante la confidencialidad de la información censal a nivel de hogar. Se utilizaron en el análisis distancias euclídeas planas en lugar de distancias por ruteo, como se acostumbra últimamente en la bibliografía, para tener un dato más preciso. Esta decisión se basó en que el sorteo de puntos aleatorios para completar la capa de domicilios y el sorteo también aleatorio para muestrear esa capa, incorporan una variabilidad que hace innecesaria tal precisión en el cálculo de distancia.

En comparación con técnicas que utilizan otras fuentes de datos para reescalar la información censal (Balk et al. 2006; CIESIN, 2016), nuestra propuesta utiliza la distribución de los hogares directamente, en lugar de un proxy de esta variable como la estructura de la malla urbana o la cobertura de suelo. Dado que ninguna de las fuentes de información disponibles que podían proveer la distribución espacial de los hogares tenía cobertura nacional y que cada fuente distribuía en forma diferente sus errores, la combinación de las fuentes se realizó a una escala de mayor detalle al que iba a ser utilizada (Botto y Detomasi, 2015).

La metodología aplicada difiere de otras publicadas en la combinación de tres puntos: el modelado de la demanda a nivel de puntos re-escalando la información censal, la exploración de localidades no prefijadas para la ubicación de los servicios y la programación de un algoritmo específico y de uso libre para la aplicación de la metodología a otros problemas.

(c) Los autores 
Detomasi Araujo R. A., Botto Nuñez G. (2017): “Evaluación espacial de servicios de educación inicial: la densificación de la oferta para niños de tres años en la Administración Nacional de Educación Pública (ANEP)”, GeoFocus (Artículos), $n^{o}$ 20, p. 49-61. ISSN: 1578-5157 http://dx.doi.org/10.21138/GF.508

Softwares y algoritmos como el Flowmap, permiten hallar ubicaciones óptimas entre un conjunto predefinido de opciones (Fuenzalida 2011). La exploración de sitios no prefijados permite definir un centro teórico y que la búsqueda del punto exacto se haga en función de las posibilidades de gestión. Más aún, algoritmos iterativos como el presentado aquí permiten incorporar los nuevos centros a medida que se van confirmando, para reelaborar el análisis y ajustarlo a las decisiones ya tomadas. Esta característica lo hace más adecuado para su uso cotidiano en gestión. Aun tomando una decisión no óptima en un paso, se puede ajustar el resto del análisis para elegir la mejor ubicación para los siguientes servicios. En nuestro caso, la distancia considerada óptima exigía una escala para la distribución de la demanda que justifica el uso de distribución por puntos. En particular, como el análisis abarca todo el país y las estructuras de datos censales varían ampliamente en escala geográfica entre zonas urbanas y rurales, no correspondía el uso de manzanas o segmentos censales. Esto obligó al diseño de una metodología para re escalar los datos, que incluyó el agregado de puntos aleatorios. Estos puntos aleatorios son los que impiden el uso de distancias por ruteo. Otra opción a las distancias por ruteo o las distancias lineales, son las isócronas, por ejemplo, utilizadas por Rodríguez y colaboradores (2016) para un ejemplo en Chile. Sin embargo, a la escala trabajada en nuestro caso, presentan el mismo problema que las distancias por ruteo.

Finalmente, la metodología propuesta para la planificación de nuevos centros permite crear un conjunto de centros cuya localización y número de cupos ofrecidos satisface los criterios de accesibilidad propuestos. Si bien es poco probable que tal lista pueda ser implementada directamente y en forma estricta, provee un modelo que puede dirigir las decisiones operativas.

Teniendo en consideración los factores antedichos, es posible replicar la metodología a diversos niveles de gobierno y adaptarla para otros procesos similares. En particular, el proceso permite focalizar las políticas hacia grupos especialmente vulnerables, representados en este caso por aquellos hogares incluidos en un programa centralizado de gobierno de transferencias monetarias. Los resultados obtenidos, presentados a las instituciones ejecutoras en forma de visualizadores interactivos, se convierten en una herramienta importante a la hora de tomar decisiones puntuales sobre ubicación de futuros servicios y para la evaluación continua de las políticas de ampliación implementadas. Considerando la priorización por vulnerabilidad socioeconómica se obtuvo una cantidad de centros a construir muy cercana a la prevista por los fondos disponibles. La aplicación de este proceso implica una efectiva re-orientación de la inversión. Como ejemplo de esto, ya se ha trabajado, con una propuesta de aperturas para CAIF (Botto y Detomasi, 2015), en pos de la cobertura de los niños de 1 y 2 años que han pasado a ser su foco de atención, en la rediagramación de la Educación Inicial de Uruguay, en manos del SNIC. 
Detomasi Araujo R. A., Botto Nuñez G. (2017): "Evaluación espacial de servicios de educación inicial: la densificación de la oferta para niños de tres años en la Administración Nacional de Educación Pública (ANEP)”, GeoFocus (Artículos), $n^{o}$ 20, p. 49-61. ISSN: 1578-5157 http://dx.doi.org/10.21138/GF.508

\section{Referencias bibliográficas}

Amarante, V., de Melo, G., Vigorito, A. (2008) Metodología para la selección de los beneficiarios del nuevo sistema de Asignaciones Familiares. Informe final, Convenio UdelaR-MIDES. Disponible en: https://tinyurl.com/y9dmq6zy (Acceso: 20-09-2016)

Bai, H., Carrasco, P., Colafranceschi, M. (2014): Aplicación del Índice de Carencias Críticas al Censo y análisis del vínculo con NBI. Montevideo, MIDES. Disponible en: https://tinyurl.com/y8svf85w (Acceso: 20-09-2016)

Balk D.L., Deichmann U., Yetman G., Pozzi F., Hay S.I., Nelson A. (2006): "Determining global population distribution: methods, applications and data". Advances in Parasitology 62, 119-156. DOI: $10.1016 / \mathrm{S} 0065-308 \mathrm{X}(05) 62004-0$

Bracken, I. y Martin, D. (1989): “The generation of spatial population distributions from census centroid data", Environment and Planning A. 21, pp. 537-543. DOI: 10.1068/a210537

Bracken, I. y Martin, D. (1995): "Linkage of the 1981 and 1991 UK Censuses using surface modelling concepts", Environment and Planning A 27(3), pp. 379- 390. DOI: 10.1068/a270379

Bosque, J. y Moreno, A. (2004): Sistemas de Información Geográfica y localización de instalaciones y equipamientos. Madrid. Ra-Ma. $353 \mathrm{pp}$.

Botto G. y Detomasi, R. (2015): Bases metodológicas para la planificación espacial de servicios de educación inicial en Uruguay. Jornadas Argentinas de Geotecnologías: Trabajos completos. Pp. 121-128. Universidad Nacional de Luján - Sociedad de Especialistas Latinoamericanos en Percepción Remota - Universidad Nacional de San Luis.

Cabella, W., Filgueira, F., Giusti, A. y Macadar, D. (2012): Informe de la Comisión Técnica Honoraria para la evaluación del Censo Uruguay 2011. Instituto Nacional de Estadística.

Disponible en: https://tinyurl.com/ycdph2n3 (Acceso: 20-09-2016)

Center for International Earth Science Information Network - CIESIN - Columbia University. (2016): Documentation for the Gridded Population of the World, Version 4 (GPWv4). Palisades NY: NASA Socioeconomic Data and Applications Center (SEDAC). DOI: 10.7927/H4D50JX4

Departamento Análisis y Estudios Sociales - Dirección Nacional de Evaluación y Monitoreo Ministerio de Desarrollo Social (2015): “QQué es el Índice de Carencias Críticas? Serie de documentos", Aportes a la conceptualización de la pobreza y la focalización de las políticas sociales en Uruguay. Montevideo, MIDES. Disponible en: https://tinyurl.com/y7rqzlws (Acceso: 20-09-2016)

Detomasi, R y Botto, G. (2017) EPP: Evaluation of Proximity Programs ( $R$ Package). DOI: 10.5281/zenodo.824283

Fuenzalida Díaz, M. (2011) "Diseño de esquemas de localización óptima para hospitales del servicio de salud Viña del Mar-Quillota (Chile) discriminando según status socio-económico". GeoFocus Revista Internacional de Ciencia y Tecnología de la Información Geográfica 11:409430.

Gotelli, N.J. y Ellison, A.M. (2004): A primer of Ecological Statistics. Massachusetts, Sinauer Associates Inc. Publishers. Sunderland. 
Detomasi Araujo R. A., Botto Nuñez G. (2017): "Evaluación espacial de servicios de educación inicial: la densificación de la oferta para niños de tres años en la Administración Nacional de Educación Pública (ANEP)”, GeoFocus (Artículos), $n^{o}$ 20, p. 49-61. ISSN: 1578-5157 http://dx.doi.org/10.21138/GF.508

INE (2012): Resultados del Censo de Población 2011: población, crecimiento y estructura por sexo y edad. Instituto Nacional de Estadística. Disponible en: https://tinyurl.com/ybauy37y (Acceso: 2009-2016)

INE (s/f): Unidades Geoestadísticas (U-Geo)-Uruguay. Instituto Nacional de Estadística. Disponible en: https://tinyurl.com/ycx8emta (Acceso: 20-09-2016)

Li, T., Pullar, D. V., Corcoran, J. y Stimson, R. J. (2007): “A comparison of spatial disaggregation techniques as applied to population estimation for south east Queensland (SEQ), Australia", Applied GIS, 3 (9), pp. 1-16.

Maantay, J.A., Maroko, A.R. y Herrmann, C. (2007): "Mapping population distribution in the urban environment: the cadastral-based expert dasymetric system (CEDS)", Cartography and Geographic Information Science, 34 (2), pp. 77-102. DOI: 10.1559/152304007781002190

Matallana Villareal, J. (2010). Equidad espacial en el acceso a la educación incial en Bogotá. Bogotá: Ediciones Uniandes.

Okabe, A., Boots, B., Sugihara, K. and Chiu, S. (2000): Spatial Tessellations: Concepts and Applications of Voronoi Diagrams. Chichester, 2nd ed. Wiley.

R Core Team (2015): R: A language and environment for statistical computing. Vienna, Austria. $\mathrm{R}$ Foundation for Statistical Computing, ISBN 3-900051-07-0. Disponible en: http://www.rproject.org/ (Acceso: 20-09-2016)

Ramírez, L. y Bosque, J. (2001): "Localización de hospitales: Analogías y diferencias del uso del modelo p-mediano en SIG raster y vectorial", Anales de Geografía de la Universidad Complutense, 21, Madrid, pp. 53-79.

Rodríguez, P.; Valenzuela, J. P.; Suchan, Karol; Truffello, Ricardo; Norel, Nicol (2016) Determinando el acceso real de los estudiantes a establecimientos educacionales efectivos para generar políticas públicas que mejoren la provisión de educación de calidad. Documento de trabajo CIAE No19, Universidad de Chile. Disponible en: https://tinyurl.com/yceal7o7 (Acceso: 2009-2016)

Sen, A. (1996). "Capacidad y bienestar." En: Nussbaum M. C. \& Sen A. (Comps.), La calidad de vida. México: Fondo de Cultura Económica. 54-83.

UNESCO Sector Educación. (2011) Plan Nacional de Educación 2010-2030 (Componente ANEP): aportes para su elaboración. Montevideo: UNESCO-ANEP-OPP. 178 p. Disponible en: https://tinyurl.com/y96ybrct (Acceso: 20-09-2016) 
